\title{
Incidence and management of pyrotinib-associated diarrhea in HER2-positive advanced breast cancer patients
}

\author{
Chen Fang ${ }^{1 *}$, Jiahuai Wen ${ }^{1 *}$, Mengling Kang ${ }^{1}$, Yuzhu Zhang ${ }^{2}$, Qianjun Chen ${ }^{1}$, Liping Ren ${ }^{1}$ \\ ${ }^{1}$ Department of Breast Oncology, Guangdong Hospital of Traditional Chinese Medicine, Guangzhou, China; ${ }^{2}$ Department of Breast Oncology, \\ Shuguang Hospital of Shanghai University of Traditional Chinese Medicine, Shanghai, China \\ Contributions: (I) Conception and design: C Fang; (II) Administrative support: L Ren; (III) Provision of study materials or patients: Q Chen; (IV) \\ Collection and assembly of data: M Kang, J Wen; (V) Data analysis and interpretation: Y Zhang; (VI) Manuscript writing: All authors; (VII) Final \\ approval of manuscript: All authors. \\ \#These authors contributed equally to this work. \\ Correspondence to: Liping Ren. Department of Breast Oncology, Guangdong Hospital of Traditional Chinese Medicine, 111 Dade Road, Guangzhou \\ 510060, China. Email: rlp00827@sina.com.
}

\begin{abstract}
Background The PHOEBE study showed that pyrotinib is an alternative treatment option for patients with human epidermal growth factor receptor 2 (HER2)-positive advanced breast cancer after trastuzumab and chemotherapy. Diarrhea was a common adverse event that could compromise or modify treatment administration.

Methods: We conducted a retrospective cohort study of HER2-positive advanced breast cancer patients receiving a pyrotinib-based regimen between August 2018 and January 2021. The risk of diarrhea with 95\% confidence limits (CLs) was calculated and management was analyzed.

Results: A total of 46 HER2-positive advanced breast cancer patients were enrolled. Diarrhea of any grade occurred in $100 \%$ of patients, and grade 3 diarrhea was observed in $52.2 \%(n=24)$ of patients. Treatment dose modification was implemented in $39.1 \%(n=18)$ of advanced breast cancer patients. Compared with other treatment regimens, patients receiving pyrotinib plus vinorelbine suffered the highest risk of diarrhea (60\%), though no significant difference was confirmed $(\mathrm{P}=0.77)$. Antidiarrheal agents were commonly used and loperamide-based regimens achieved an ideal antidiarrheal effect (95.3-100\%), while non-loperamidebased regimens (such as montmorillonite powder) had a lower control rate (78.6\%).
\end{abstract}

Conclusions: Diarrhea was a common adverse event of pyrotinib-based treatment, and approximately $50 \%$ of patients suffered high grade diarrhea. Loperamide-based antidiarrheal agents could commendably manage most episodes.

Keywords: Breast cancer; pyrotinib; diarrhea

Submitted Nov 18, 2021. Accepted for publication Jan 18, 2022.

doi: 10.21037/apm-21-3978

View this article at: https://dx.doi.org/10.21037/apm-21-3978

\section{Introduction}

Pyrotinib is a second-generation, irreversible, panhuman epidermal growth factor receptor (HER) tyrosine kinase inhibitor (TKI) targeting epidermal growth factor receptor (EGFR), HER2, and human epidermal growth factor receptor 4 (HER4) (1). Phase II studies demonstrated that pyrotinib plus capecitabine significantly improved the overall response rate and progression-free survival (PFS) of HER2-positive advanced breast cancer patients previously treated with taxanes, anthracyclines, and/or trastuzumab (2). The PHOEBE trial indicated that, compared with lapatinib plus capecitabine, secondline pyrotinib plus capecitabine obtained a median PFS of 5.7 months and an objective response rate (ORR) of $67.2 \%$ (3). Based on the above clinical trial, the Breast Cancer Guidelines of the Chinese Society of Clinical 
Oncology (CSCO) recommend a pyrotinib-based regimen as second-line treatment after trastuzumab treatment failure for HER2-positive advanced breast cancer patients.

Diarrhea is a common adverse event during anti-HER2 treatment. CLEOPATRA study indicated that $23 \%$ of breast cancer patients receiving pertuzumab and trastuzumab suffered diarrhea, and most cases were grade 1-2 events. However, diarrhea is the most common TKI-related adverse event (4), and the overall incidence of diarrhea was reported to be as high as $96.9 \%$ in phase II study (2). While grade 1-2 diarrhea can influence quality of life, grade 3-4 diarrhea can cause dehydration and electrolyte disturbance, which may be life threatening in severe cases (5). Therefore, diarrhea prevention and intervention are essential for patients receiving pyrotinib-based treatment (6). So far, no study focused on the occurrence risk and treatment of pyrotinib-associated diarrhea.

In the present study, we retrospectively collected the data of advanced breast cancer patients receiving pyrotinib and evaluated the level of diarrhea, aiming to evaluate the effect of various medical interventions on drug-related diarrhea. We present the following article in accordance with the STROBE reporting checklist (available at https://apm. amegroups.com/article/view/10.21037/apm-21-3978/rc).

\section{Methods}

\section{Study population}

Advanced breast cancer patients from August 2018 to January 2021 at Guangdong Hospital of Traditional Chinese Medicine were retrospectively reviewed. Enrolled patients were required to meet the following inclusion criteria: (I) pathologically and/or radiographically confirmed metastasis; (II) received pyrotinib-based treatment; (III) HER2 positive, defined as immunohistochemistry $3+$ and/ or fluorescence in situ hybridization positive with $\geq 2$-fold amplification; (IV) female. The exclusion criteria were as follows: (I) concomitant non-mammary malignancy; (II) preexisting gastrointestinal disease that could induce diarrhea (such inflammatory bowel disease, Crohn's disease, ulcerative colitis); (III) not enough data could be extracted.

All procedures performed in this study involving human participants were in accordance with the Declaration of Helsinki (as revised in 2013). The study was approved by institutional ethics board of Guangdong Hospital of Traditional Chinese Medicine (No. ZF2020-274-01). Written informed consent was obtained from each participant before inclusion in this study.

\section{Data collection}

Clinicopathological data were collected for each participant, such as age and metastatic sites. Salvage treatment information was also collected, including: (I) pyrotinib initial dose, modified dose, and treatment duration; (II) combined chemotherapy and anti-HER2 agent; (III) lines of therapy, number of prior lines of therapy, and details of drug-related diarrhea (occurrence time, duration, severity, intervention treatment, and therapeutic effect). All patients were followed up in the out-patient department.

The severity of diarrhea was evaluated based on the National Cancer Institute-Common Terminology Criteria for Adverse Events (NCI-CTC 4.0) (7). Grade 1: Increase of $<4$ stools per day over baseline; mild increase in ostomy output compared to baseline. Grade 2: Increase of 4-6 stools per day over baseline; moderate increase in ostomy output compared to baseline; limited instrumental activities of daily living (ADL). Grade 3: Increase of $\geq 7$ stools per day over baseline; hospitalization required; severe increase in ostomy output compared to baseline; limited self-care ADL. Grade 4: Life-threatening consequences; urgent intervention required.

\section{Statistical analysis}

The Student's $t$-test or Mann-Whitney U test was performed for comparisons of continuous variables, and categorical variables were compared using the $\chi^{2}$ test or Fisher's exact test. The primary endpoint was the occurrence risk of pyrotinib-related diarrhea, which was compared by the independent samples $t$-test. Subgroup analyses were performed to compare the efficacy of various antidiarrheal interventions. Multiple regression analysis was used to minimize the relevant bias. SPSS 23.0 (IBM, New York) was used for all analyses, and two-tailed $\mathrm{P} \leq 0.05$ was considered as statistically significant.

\section{Results}

\section{Clinicopathological characteristics of the study participants}

A total of 46 HER2-positive advanced breast cancer patients were enrolled in the present study (Figure 1), and Table 1 shows the baseline clinicopathological characteristics. The mean age was 52.5 (range, 25-83) years and average follow- 


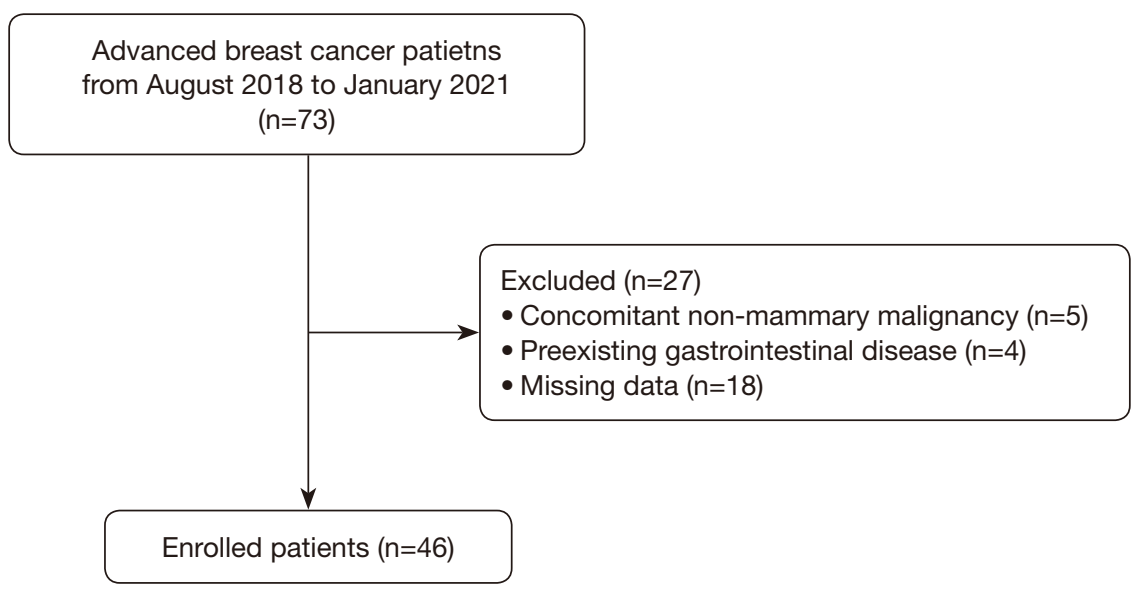

Figure 1 Patient selection flow chart.

up time was 5.4 months. Fourteen (30.4\%) patients had local recurrence, soft tissue metastasis, or bone metastasis, while other patients $(69.6 \%)$ had visceral metastases. The initial dose of pyrotinib was $400 \mathrm{mg}$ daily, and $39.1 \%$ $(n=18)$ of patients experienced dose modification due to an adverse event (12 patients: $320 \mathrm{mg}$ daily, 6 patients: $280 \mathrm{mg}$ daily). Analyzing the therapeutic regimen, 2 (4.3\%) patients received pyrotinib only, while $95.7 \%$ of participants received combined therapy. Forty-four patients received adjuvant chemotherapy (capecitabine, $n=30$; antimicrotubule drugs, $n=14)$. Moreover, 6 patients $(13.0 \%)$ synchronously received trastuzumab.

\section{Pyrotinib-related diarrbea}

All patients developed different grades of diarrhea 1-7 days after the first pyrotinib administration (average time $2.76 \pm 2.17$ days). Four patients $(8.7 \%)$ experienced $<1$ week of diarrhea, 19 patients (41.3\%) had diarrhea lasting 1 week to 1 month, and $30.4 \%$ of patients suffered more than 3 months of diarrhea. Based on the NCI-CTC 4.0 criteria, 10 patients $(21.7 \%)$ suffered grade 1 diarrhea and 12 patients (26.1\%) suffered grade 2 diarrhea. Grade 3 diarrhea occurred in $52.2 \%$ of patients and no grade 4 diarrhea was reported. Only a few cases required hospitalization due to uncontrolled diarrhea.

Four hundred milligram of Pyrotinib caused $80 \%$ of grade 3-4 diarrhea occurrence without antidiarrheal treatment. However, under identical antidiarrheal intervention, the pyrotinib dosage levels were not significantly related to the incidence rate of diarrhea $(\mathrm{P}=0.14)$. Approximately $66.0 \%$ of patients among $240 \mathrm{mg}$ pyrotinib and $320 \mathrm{mg}$ pyrotinib subgroups and $57.1 \%$ of patients among $400 \mathrm{mg}$ pyrotinib subgroup experienced grade 1 diarrhea, while $320 \mathrm{mg}$ and $400 \mathrm{mg}$ pyrotinib induced $14.3-16.7 \%$ risk of grade 2 diarrhea. Among advanced breast cancer patients receiving chemotherapy plus pyrotinib, vinorelbine plus pyrotinib induced the highest incidence rate of grade 3 diarrhea (60\%). However, no significant difference was shown between various chemotherapy regimens $(\mathrm{P}=0.77)$.

\section{Interventions for diarrbea}

Loperamide was the main medication used for diarrhea treatment. Twenty-one patients (45.7\%) received loperamide only, 8 patients $(17.4 \%)$ received loperamide plus montmorillonite powder, and 3 patients $(6.5 \%)$ used loperamide and montmorillonite powder plus probiotics for diarrhea treatment. We classified the antidiarrheal effect as effective (no diarrhea), partially effective (NCICTC diarrhea grade decreased by 1 grade or more), and ineffective (diarrhea was not changed or worsened). Based on the medication, the effective rates of the loperamide only group and loperamide-combined group were $95.3 \%$ and $100 \%$, respectively, while the non-loperamide group only had a $78.6 \%$ effective rate $(\mathrm{P}=0.26)$.

Based on the antidiarrheal drug intervention, patients were divided into the loperamide only group (group A), loperamide combined with other antidiarrhea drugs group (group B), and non-loperamide group (group C). The total effective rate was $95.3 \%$ in group A, $100 \%$ in group B, and $78.6 \%$ in group C. The drug intervention plan and efficacy are shown in Table 2. However, there was no significant 
Table 1 Baseline demographic and disease characteristics of the 46 patients

\begin{tabular}{|c|c|}
\hline Items & Data \\
\hline \multicolumn{2}{|l|}{ Age } \\
\hline Median/years & 52.5 \\
\hline Range/years & $25-83$ \\
\hline$<65$ years, $\mathrm{n}(\%)$ & $44(95.7)$ \\
\hline$\geq 65$ years, $\mathrm{n}(\%)$ & $2(4.3)$ \\
\hline \multicolumn{2}{|l|}{ Sites of metastases, $\mathrm{n}(\%)$} \\
\hline Visceral & $32(69.6)$ \\
\hline Liver & $16(34.8)$ \\
\hline Lung & $20(43.5)$ \\
\hline Brain & $7(15.2)$ \\
\hline Non-visceral & $14(30.4)$ \\
\hline \multicolumn{2}{|l|}{ Chemotherapy regimens, n (\%) } \\
\hline Capecitabine & $30(65.2)$ \\
\hline Vinorelbine & $10(21.7)$ \\
\hline Albumin-bound paclitaxel & $2(4.3)$ \\
\hline Docetaxel & $2(4.3)$ \\
\hline Pyrotinib only & $2(4.3)$ \\
\hline \multicolumn{2}{|c|}{ Combine anti-HER2 therapy, n (\%) } \\
\hline Trastuzumab & $6(13.0)$ \\
\hline None & $40(87.0)$ \\
\hline \multicolumn{2}{|c|}{ Lines of treatment in the metastatic setting, $\mathrm{n}(\%)$} \\
\hline 1 & $1(2.2)$ \\
\hline 2 & $14(30.4)$ \\
\hline 3 & $16(34.8)$ \\
\hline 4 & 13 (28.3) \\
\hline 5 & $2(4.3)$ \\
\hline
\end{tabular}

difference in antidiarrheal efficacy among all groups $(\mathrm{P}=0.26)$.

\section{Discussion}

Anti-HER2 agents are essential regimens for HER2positive breast cancer. Trastuzumab and pertuzumab are the first-line preferred recommendation based on the NCCN guideline, with approximately $28.1 \%$ of grade 1 or 2 diarrhea occurrence risk. However, if trastuzumab occurred, TKI becomes essential medication for subsequent treatment. Pyrotinib is second-generation, irreversible TKI, and diarrhea and hand-foot syndrome were the most common adverse events, and grade 3 or worse adverse events were reported for $31 \%$ and $16 \%$ patients respectively as reported in PHOBE study (3). Severe diarrhea can lead to dose reduction or treatment interruption, which might weaken the curative effect. Most TKIs require oral administration daily for a certain period, and even grade 1-2 diarrhea can significantly affect quality of life. It is essential for clinicians to manage and treat pyrotinib-related diarrhea. The mechanism of pyrotinib-related diarrhea remains unclear (5), though common hypotheses are as follows: (I) the inhibition of the EGFR signaling pathway can hamper intestinal epithelial cell proliferation (8), the transportation of nutrients and electrolytes (9), and the expression of brush border peptidases (10), causing intestinal mucosal atrophy; (II) dysregulation of the EGFR signaling pathway can lead to excessive accumulation of chloride; (III) disruption of gastrointestinal microcirculation or inflammatory responses can cause diarrhea. However, since the occurrence mechanism remains unclear, preventive treatment before TKI administration is not well established.

Previous clinical trials have indicated that pyrotinib is associated with a high incidence of diarrhea. In a phase I trial investigating the efficacy of pyrotinib plus capecitabine (11), the incidence rates of diarrhea of any grade and grade $3 / 4$ were $85.7 \%$ and $10.7 \%$, respectively. In the subsequent phase II trial (3), the incidence rates of any grade of diarrhea in the pyrotinib group and control group were $96.9 \%$ and $44.4 \%$, respectively, while the incidence rate of grade $3 / 4$ diarrhea was $15.4 \%$ in the pyrotinib group and $4.8 \%$ in the control group. Similarly, $98.4 \%$ of participants suffered various grades of diarrhea and $30.8 \%$ had grade $\geq 3$ diarrhea in the phase III PHOENIX trial (1). Furthermore, a previous study also found that grade $\geq 3$ diarrhea usually occurred in the first treatment cycle, and the grade improved in subsequent treatment cycles. In a real world study, the incidence of any grade pyrotinibrelated diarrhea was reported as $98.2 \%$, while the incidence of grade $\geq 3$ diarrhea was $21.8 \%$ (12).

In the present study, no significant association between diarrhea occurrence risk, severity, and chemotherapy/ other anti-HER2 regimens was demonstrated. Moreover, the actual occurrence rates of all-grade and grade 3/4 diarrhea were $100 \%$ and $54.8 \%$ respectively, which were higher than those reported in previous clinical trials. The possible reason may be that many patients did not initiate 
Table 2 Comparison of clinical efficacy in the 3 groups (n, \%)

\begin{tabular}{|c|c|c|c|}
\hline Classification & Group A $(n=21)$ & Group B $(n=11)$ & Group C $(n=14)$ \\
\hline Partial responders (reduction in diarrhea by $1 \mathrm{NCl}-\mathrm{CTC}$ grade) & $14(66.7)$ & $9(81.8)$ & $7(50.0)$ \\
\hline Non-responders (no change in diarrhea) & $1(4.8)$ & $0(0.0)$ & $3(21.4)$ \\
\hline
\end{tabular}

$\mathrm{NCl}-\mathrm{CTC}$, National Cancer Institute-Common Terminology Criteria for Adverse Events.

antidiarrheal intervention based on the recommended time, and some patients even rejected the proper medical intervention. This phenomenon indicates that the standard treatment for pyrotinib-related diarrhea is not promoted in clinical practice, and oncologists should emphasize the proper management of this adverse event during pyrotinib treatment.

The onset of diarrhea occurs mostly within 1 week after the first dose of pyrotinib, and the average time was 2.86 days. A total of $45.2 \%$ of patients suffered 1 week to 1 month of diarrhea. The above results are consistent with previous reports on TKIs (13). Diarrhea prevention and early intervention are essential to improve patient compliance. In previous clinical studies, dose modification or treatment termination due to diarrhea were rare. In the present study, $40.5 \%$ of patients received dose modification and $14.3 \%$ of patients received the lowest dose level at $240 \mathrm{mg}$ daily. Even no PFS difference was proven among various pyrotinib-dose subgroups $(\mathrm{P}=0.48)$, good management of pyrotinib-related diarrhea was important to prevent dose reduction in clinical practice, which influences the therapeutic effect.

The 2019 EGFR-TKI ADR Management Chinese Expert Consensus recommended that loperamide, montmorillonite, and probiotics are the preferred treatments for TKI-associated diarrhea, while codeine (30 mg BID) could be added in severe cases (14). In the present study, we classified patients into 3 groups: loperamide only group (group A), loperamide combined with other antidiarrheal drugs group (group B), and nonloperamide group (group C). The results indicated that loperamide-based regimens achieved a high control rate of diarrhea (95.3-100\%), while non-loperamide treatment had an inferior antidiarrheal effect (78.6\%). This suggests that loperamide is an essential treatment for pyrotinib-related diarrhea. On the basis of loperamide, the addition of other antidiarrheal agents (e.g., montmorillonite, probiotics) could not significantly improve the control rate, which might be due to the limited number of enrolled patients in the study. In this study, a substantial portion of patients did not receive standardized treatment according to the recommended time of diarrhea occurrence, and even rejected drug intervention. This was the possible reason for the high incidence rate of diarrhea at all grades in this study. Thus, rational treatment for pyrotinib-related diarrhea should be promoted in clinical practice.

In 2021, the CSCO Breast Cancer Expert Committee published the expert consensus on the management of adverse events of ErbB family TKIs in breast cancer (15). For TKI-related diarrhea, the recommended treatment for grade 1-2 diarrhea without complications is water and electrolyte replenishment and loperamide. If the symptoms do not improve, octreotide, opioids, and treatment interruption can be recommended until the diarrhea level is downgraded to grade 1, and the original dose of pyrotinib should be recommenced on the basis of loperamide. However, TKIs and chemotherapy should be suspended when diarrhea of any grade with complications or grade 3-4 diarrhea occur. Atropine and codeine can be added to loperamide for antidiarrhea treatment. If symptoms are relieved, TKIs can be recommenced at a decreased dose. Furthermore, regular fecal examination and fecal culture should be performed to exclude infectious diarrhea that requires antibiotic treatment (16). In terms of daily care, a low-fat and lowfiber diet is recommended, and caffeine, alcohol, dairy products, orange juice, grape juice, and spicy food should be avoided (14). Additionally, several new treatments are currently under investigation (17). Ianiro et al. found that fecal microbiota transplantation (FMT) could relieve diarrhea symptoms caused by TKIs in metastatic renal cell carcinoma patients, and FMT from healthy donors achieves a better effect than placebo FMT (18). The above research indicates that FMT may become a new therapeutic approach for TKI-associated diarrhea.

Prevention is more important than intervention among breast cancer patients receiving pyrotinib. At present, there is no clinical study focusing on the primary prevention 
of pyrotinib-related diarrhea. The Consensus of Adverse Reactions to Tyrosine Kinase Inhibitors in Breast Cancer suggests that if patients suffer interruption to pyrotinib treatment caused by grade $\geq 3$ diarrhea, preventive loperamide should be given simultaneously with pyrotinib recommencement.

This study had several limitations. First, because of the recent approval of the clinical application of pyrotinib, the number of patients meeting the inclusion criteria was small, which partly influenced the statistical analyses. Second, the bloody concentration of pyrotinib could not be monitored in the observational study, leading to the deficiency of pharmacokinetics analysis of pyrotinib. Third, subgroup analyses based on combined therapy, antidiarrheal agents, and survival analyses were not available due to the limited number enrolled patients.

In conclusion, diarrhea is the most common adverse event of pyrotinib. The high incidence rate and severity greatly lower the quality of life of patients. Thus, effective and standardized management of pyrotinib-related diarrhea is essential, which may prevent treatment interruption, dose modification, and may improve patient compliance and the therapeutic effect. Further researches with larger number of participants are needed to comprehensively develop effective strategy for pyrotinib-related diarrhea prevention.

\section{Acknowledgments}

Funding: None.

\section{Footnote}

Reporting Checklist: The authors have completed the STROBE reporting checklist. Available at https://apm. amegroups.com/article/view/10.21037/apm-21-3978/rc

Data Sharing Statement: Available at https://apm.amegroups. com/article/view/10.21037/apm-21-3978/dss

Conflicts of Interest: All authors have completed the ICMJE uniform disclosure form (available at https://apm. amegroups.com/article/view/10.21037/apm-21-3978/coif). The authors have no conflicts of interest to declare.

Ethical Statement: The authors are accountable for all aspects of the work in ensuring that questions related to the accuracy or integrity of any part of the work are appropriately investigated and resolved. All procedures performed in this study involving human participants were in accordance with the Declaration of Helsinki (as revised in 2013). The study was approved by institutional ethics board of Guangdong Hospital of Traditional Chinese Medicine (No. ZF2020-274-01). Written informed consent was obtained from each participant before inclusion in this study.

Open Access Statement: This is an Open Access article distributed in accordance with the Creative Commons Attribution-NonCommercial-NoDerivs 4.0 International License (CC BY-NC-ND 4.0), which permits the noncommercial replication and distribution of the article with the strict proviso that no changes or edits are made and the original work is properly cited (including links to both the formal publication through the relevant DOI and the license). See: https://creativecommons.org/licenses/by-nc-nd/4.0/.

\section{References}

1. Li X, Yang C, Wan H, et al. Discovery and development of pyrotinib: A novel irreversible EGFR/HER2 dual tyrosine kinase inhibitor with favorable safety profiles for the treatment of breast cancer. Eur J Pharm Sci 2017;110:51-61.

2. Ma F, Ouyang Q, Li W, et al. Pyrotinib or Lapatinib Combined With Capecitabine in HER2-Positive Metastatic Breast Cancer With Prior Taxanes, Anthracyclines, and/or Trastuzumab: A Randomized, Phase II Study. J Clin Oncol 2019;37:2610-9.

3. Xu B, Yan M, Ma F, et al. Pyrotinib plus capecitabine versus lapatinib plus capecitabine for the treatment of HER2-positive metastatic breast cancer (PHOEBE): a multicentre, open-label, randomised, controlled, phase 3 trial. Lancet Oncol 2021;22:351-60.

4. National Cancer Institute. Common terminology criteria for adverse events. 2018. p. v5-8. Available online: https://ctep.cancer.gov/protocoldevelopment/ electronic_applications/docs/CTCAE_v5_Quick_ Reference_8.5x11.pdf

5. Hirsh V, Blais N, Burkes R, et al. Management of diarrhea induced by epidermal growth factor receptor tyrosine kinase inhibitors. Curr Oncol 2014;21:329-36.

6. Cherny NI. Evaluation and management of treatmentrelated diarrhea in patients with advanced cancer: a review. J Pain Symptom Manage 2008;36:413-23.

7. Basch E, Reeve BB, Mitchell SA, et al. Development of the National Cancer Institute's patient-reported 
outcomes version of the common terminology criteria for adverse events (PRO-CTCAE). J Natl Cancer Inst 2014;106:dju244.

8. Berlanga-Acosta J, Playford RJ, Mandir N, et al. Gastrointestinal cell proliferation and crypt fission are separate but complementary means of increasing tissue mass following infusion of epidermal growth factor in rats. Gut 2001;48:803-7.

9. Opleta-Madsen K, Hardin J, Gall DG. Epidermal growth factor upregulates intestinal electrolyte and nutrient transport. Am J Physiol 1991;260:G807-14.

10. Goodlad RA, Raja KB, Peters TJ, et al. Effects of urogastrone-epidermal growth factor on intestinal brush border enzymes and mitotic activity. Gut 1991;32:994-8.

11. Li Q, Guan X, Chen S, et al. Safety, Efficacy, and Biomarker Analysis of Pyrotinib in Combination with Capecitabine in HER2-Positive Metastatic Breast Cancer Patients: A Phase I Clinical Trial. Clin Cancer Res 2019;25:5212-20.

12. Li F, Xu F, Li J, et al. Pyrotinib versus trastuzumab emtansine for HER2-positive metastatic breast cancer after previous trastuzumab and lapatinib treatment: a real- world study. Ann Transl Med 2021;9:103.

13. Rugo HS, Di Palma JA, Tripathy D, et al. The characterization, management, and future considerations for ErbB-family TKI-associated diarrhea. Breast Cancer Res Treat 2019;175:5-15.

14. EGFR-TKI ADR Management Chinese Expert Consensus. Zhongguo Fei Ai Za Zhi 2019;22:57-81.

15. Wang BY, Ge R, Jiang ZF. Expert consensus on the management of adverse events of ErbB family tyrosine kinase inhibitors in breast cancer. Zhonghua Zhong Liu Za Zhi 2020;42:798-806.

16. Ippoliti C. Antidiarrheal agents for the management of treatment-related diarrhea in cancer patients. Am J Health Syst Pharm 1998;5 5:1573-80.

17. Bowen JM. Mechanisms of TKI-induced diarrhea in cancer patients. Curr Opin Support Palliat Care 2013;7:162-7.

18. Ianiro G, Rossi E, Thomas AM, et al. Faecal microbiota transplantation for the treatment of diarrhoea induced by tyrosine-kinase inhibitors in patients with metastatic renal cell carcinoma. Nat Commun 2020;11:4333.
Cite this article as: Fang C, Wen J, Kang M, Zhang Y, Chen Q, Ren L. Incidence and management of pyrotinib-associated diarrhea in HER2-positive advanced breast cancer patients. Ann Palliat Med 2022;11(1):210-216. doi: 10.21037/apm-21-3978 